\title{
Structure and Catalytic Properties of Pt-Modified Hyper-Cross-Linked Polystyrene Exhibiting Hierarchical Porosity
}

\author{
Lyudmila M. Bronstein, ${ }^{*}, \uparrow$ Günter Goerigk,, Maxim Kostylev, ${ }^{\dagger}$ Maren Pink, ${ }^{\dagger}$ Irina A. Khotina, \\ Peter M. Valetsky, ${ }^{\S}$ Valentina G. Matveeva," Esther M. Sulman," Michael G. Sulman," \\ Alexei V. Bykov," Nataliya V. Lakina," and Richard J. Spontak ${ }^{\perp}$ \\ Department of Chemistry, Indiana University, Bloomington, Indiana 47405, Institut für Festkörperforschung, \\ Forschungszentrum Jülich, Postfach 1913, D-52425 Jülich, Germany, A. N. Nesmeyanov Institute of \\ Organoelement Compounds, Moscow 117813, Russia, Tver Technical University, Tver 170026, Russia, and \\ Departments of Chemical Engineering and Materials Science \& Engineering, North Carolina State University, \\ Raleigh, North Carolina 27695
}

Received: August 6, 2004

\begin{abstract}
The structural transformation and catalytic properties of metal/polymer nanocomposites derived from hypercross-linked polystyrene (HPS) exhibiting both microporosity and macroporosity, and filled with Pt nanoparticles, are investigated in the direct oxidation of L-sorbose to 2-keto-L-gulonic acid. Transmission electron microscopy, X-ray photoelectron spectroscopy, X-ray diffraction, anomalous small-angle X-ray scattering, and catalytic studies suggest that the catalytically active species, nanoparticles of mixed composition with a mean diameter of $1.6 \mathrm{~nm}$, develop after the initial induction period. At the highest selectivity (96.8\%) at $100 \%$ L-sorbose conversion, the catalytic activity is measured to be $2.5 \times 10^{-3} \mathrm{~mol} / \mathrm{mol} \mathrm{Pt}-\mathrm{s}$, which corresponds to a 4.6-fold increase in activity relative to the Pt-modified microporous HPS previously reported. This substantial increase in catalytic activity is attributed to the presence of macropores, which facilitate mass transport and, consequently, accessibility of the nanoparticle surface for reactants.
\end{abstract}

\section{Introduction}

Metalated polymer nanocomposites continue to receive considerable attention since their bulk properties can be greatly, and desirably, altered relative to those of pure polymers. ${ }^{1-4}$ Nanoparticles incorporated into a polymer system may impart magnetic, semiconducting, or catalytic properties, depending on the nanoparticle species and its characteristics. In recent years, catalysis employing metal nanoparticles has become a subject of intense interest due, in major part, to the enhanced activity and selectivity of nanostructured catalysts. ${ }^{5-7}$ When catalytic nanoparticles are formed on the surface of an inorganic or carbon substrate, however, the nanoparticle size and morphology cannot be precisely regulated. ${ }^{8,9}$ One reliable means by which to overcome this challenge is to grow the nanoparticles in nanostructured polymers exhibiting well-defined buried interfaces. ${ }^{10-12}$ Such interfaces can be conveniently generated via (i) microphase separation in solution or bulk ${ }^{13-23}$ or (ii) formation of nanopores, or nanocavities, within a polymer matrix. ${ }^{24}$ Nanopores can be designed into polymers by frustrating the chain packing of complex macromolecules. ${ }^{25}$ Conversely, nanoporosity can be induced in polymers by special treatment, such as exposure to a supercritical fluid under a select set of conditions. ${ }^{26}$ When nanoporous polymers are used as matrixes in which to grow nanoparticles, the size of the nanoparticles may be physically restricted by the pore size of the polymer.

* To whom correspondence should be addressed. E-mail: lybronst@ indiana.edu.

Indiana University.

Institut für Festkörperforschung.

$\S$ A.N. Nesmeyanov Institute of Organoelement Compounds.

" Tver Technical University.

${ }^{\perp}$ North Carolina State University.
This approach is widespread in the production of mesoporous solids wherein metal compounds are incorporated inside a porous inorganic medium so that nanoparticles can subsequently be grown within the pores. ${ }^{27,28}$ This general strategy is not typically employed for use with polymeric media, since most polymers are dense and do not consist of regular interpenetrating cavities.

The development of nanoporous polymers has been greatly stimulated by the need for materials with a low dielectric constant for next-generation microelectronics. ${ }^{29}$ Production of such materials with closed nanopores can be achieved, for instance, by templating over block copolymers possessing a body- or face-centered cubic spherical morphology and a thermally labile block. If the pores are openly connected through the use of other morphologies, the resultant polymer membrane can be surface-decorated by metal deposition, resulting in metal-polymer nanocomposites. ${ }^{24,30}$ Alternatively, delocalized solvent crazing can yield nanoporous polymers possessing interpenetrating pores that can serve as nanoreactors in the formation and stabilization of highly dispersed metal nanoparticles. Amorphous porous poly(ethylene terephthalate) prepared in this fashion has been successfully used as a matrix in which to grow metallic $\mathrm{Ni}$ nanoparticles via reduction of nickel perchlorate by sodium borohydride. ${ }^{31}$ At the reaction conditions examined, pore dimensions influence the state of the final product. Subtle control over nanoparticle size and size distribution, which are vital to ultimate property development, cannot be realized, however, with the ill-defined pores formed in a crazed polymer matrix. Nanoporous polymer microspheres containing continuous channels lined with poly(acrylic acid) have been reported by $\mathrm{Lu}$ et al. ${ }^{32}$ These microspheres are prepared by UV-cross-linking of poly(tert-butyl acrylate- $b$-2- 
SCHEME 1: Illustration of the Direct Catalytic
Oxidation of L-Sorbose to 2-Keto-L-gulonic Acid

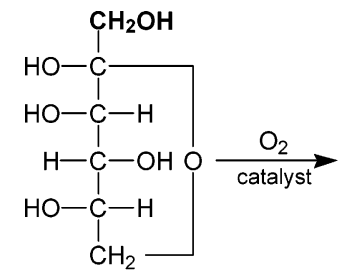

L-sorbose

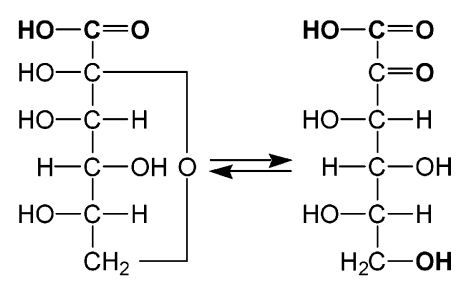

2-keto-L-gulonic acid cinnamoyloxyethyl methacrylate) micelles, followed by hydrolysis. Growth of Pd nanoparticles within the nanopores is conducted by repeatedly immersing the microspheres in $\mathrm{Pd}\left(\mathrm{NO}_{3}\right)_{2}$ (aq) solution and reducing the Pd species with hydrazine, which results in $\mathrm{Pd}$ nanoparticles varying in size and loading level.

Hyper-cross-linked polystyrene (HPS) $)^{33,34}$ is another nanoporous polymeric medium that can be considered for regulated nanoparticle growth. In this polymer network with a formal cross-link density exceeding $100 \%$, rigid nanoscale cavities measuring about $2 \mathrm{~nm}$ form spontaneously during synthesis. This material is obtained by incorporating methylene groups between adjacent phenyl rings in dissolved PS homopolymer or gelled poly(styrene-r-divinylbenzene) copolymer in the presence of ethylene dichloride, which is a good solvent for the polymer. A unique feature of HPS is its ability to swell in a wide variety of different solvents, even thermodynamically poor ones. This characteristic facilitates incorporation of various organometallic compounds into the nanostructured HPS matrix and, thus, nanoparticle formation. One of our earlier studies of metalated HPS ${ }^{35}$ describes the formation of Co nanoparticles in HPS. The regulated growth of Co nanoparticles measuring $\sim 2 \mathrm{~nm}$ in diameter over a wide range of Co concentrations is attributed to the existence of nanoscale HPS cavities, which serve to physically restrict the size of the growing nanoparticles. More recently, we have examined the formation of Pt nanoparticles in HPS. ${ }^{36}$ Impregnation of HPS with a tetrahydrofuran (THF) solution containing platinic acid $\left(\mathrm{H}_{2} \mathrm{PtCl}_{6} \cdot 6 \mathrm{H}_{2} \mathrm{O}\right)$ results in the formation of $\mathrm{Pt}(\mathrm{II})$ complexes within the nanocavities of HPS. Subsequent reduction of the complexes by $\mathrm{H}_{2}$ yields stable, relatively monodisperse $\mathrm{Pt}$ nanoparticles with a mean diameter of $1.3 \mathrm{~nm}$ (since the size of the Pt precursor, $2 \mathrm{~nm}$, is restricted by the size of the HPS nanopores). This robust metal/polymer nanocomposite exhibits exceptional properties in the catalytic oxidation of L-sorbose to 2-keto-L-gulonic acid, a vitamin C precursor (see Scheme 1), without the use of any chemical modifiers normally added to attain high reaction selectivity. In general, the catalytic activity and selectivity both depend on nanoparticle size and composition.

The principal shortcoming of this system is that the microporous nature of HPS (when pores are below $\sim 2.0-2.2 \mathrm{~nm})^{37}$ likewise limits the mass transport of reactant within the metalated HPS matrix and, hence, catalytic activity. To alleviate this disadvantage, we have elected to examine Pt metalation and subsequent L-sorbose oxidation within a commercially available variant of HPS that combines both micropores and macropores (larger than $20 \mathrm{~nm}$ in diameter). While this strategy should certainly improve mass transport during reaction, we recognize the possibility that the presence of macropores might severely jeopardize control over nanoparticle size. In this work, we report on the structure and catalytic properties of a micro/ macroporous HPS nanocomposite containing Pt nanoparticles in the direct oxidation of L-sorbose. The structural transformation of this hybrid material is followed at different reaction stages by X-ray photoelectron spectroscopy (XPS), transmission electron microscopy (TEM), X-ray diffraction (XRD), and anomalous small-angle X-ray scattering (ASAXS).

\section{Experimental Section}

Materials. The HPS was generously provided by Purolite Int. (U.K.), as Macronet MN 270/386-101/100 (hereafter designated as MN-270), and was used without further purification. According to $\mathrm{N}_{2}$ sorption measurements, the BET (Brunauer-Emmet-Teller) surface area of the unmodified HPS was $738 \mathrm{~m}^{2} / \mathrm{g}$ and its total pore volume was $0.59 \mathrm{~cm}^{3} / \mathrm{g}$. About $46 \%$ of the pore population measured in the $20-100 \mathrm{~nm}$ range, while pores $<6 \mathrm{~nm}$ accounted for $\sim 13 \%$. The mean diameter of the micropores was reported by the manufacturer to be $1.5 \mathrm{~nm}^{38}$ Both $\mathrm{H}_{2} \mathrm{PtCl}_{6} \cdot 6 \mathrm{H}_{2} \mathrm{O}$ and sodium hydrogen carbonate $\left(\mathrm{NaHCO}_{3}\right)$ were obtained from Reakhim (Moscow, Russia). Reagent-grade THF was purchased from Aldrich, whereas the L-sorbose was provided by Fluka, and both were used as received. Water was purified with a Milli-Q water purification system.

Synthesis. Compared to the method described elsewhere, ${ }^{36}$ the procedure to grow Pt nanoparticles within HPS was slightly modified to be more commercially attractive. In a typical synthesis, $0.24 \mathrm{~g} \mathrm{H}_{2} \mathrm{PtCl}_{6} \cdot 6 \mathrm{H}_{2} \mathrm{O}$ was dissolved in $6 \mathrm{~mL}$ of THF under air and added to $3 \mathrm{~g}$ of $\mathrm{MN}-270$. The suspension was continuously stirred for $10 \mathrm{~min}$ so that the solution could be absorbed by the polymer and then dried at $70-75^{\circ} \mathrm{C}$ for $24 \mathrm{~h}$. The Pt content was 1.72 wt \% by elemental analysis.

Catalysis. The oxidation reaction was conducted batchwise in a specially constructed apparatus that permits independent control over parameters such as the L-sorbose and $\mathrm{NaHCO}_{3}$ concentrations, catalyst concentration, temperature, (pure) oxygen feed rate, and stirring rate. A suspension of catalyst and L-sorbose $(100 \mathrm{~mL})$ prepared at a predetermined concentration was placed in the temperature-controlled apparatus equipped with a magnetic stir bar and reflux condenser. The rate of oxygen feed was controlled by a rotameter. An equimolar quantity of alkalizing agent $\left(\mathrm{NaHCO}_{3}\right)$ was fed to the apparatus as a single injection or continuously over $180 \mathrm{~min}$ (to maintain a constant $\mathrm{pH}$ of 7.7) using an automatic dispenser. The high stirring rates employed here ensured good mixing without diffusion limitation. Samples of the reaction mixture were periodically removed for analysis. At the end of each experiment, the catalyst was separated by filtration, and the filtrate was analyzed for the presence of L-sorbose and 2-keto-L-gulonic acid. The amount of residual L-sorbose was discerned by gas chromatography (GC) through the use of a Chrom-5 chromatograph, operated isothermally with a flame-ionization detector and glass column filled with $5 \%$ SE-30 on Chromaton N-AW. Analysis of the sodium salt of 2-keto-L-gulonic acid was performed on a MILLICHROM-5 HPLC chromatograph. Normal-phase chromatography using a $10 \mathrm{~cm}$ tungsten column characterized by a 4000 theoretical plate number was chosen for sample analysis. Separon SGX-NH $\mathrm{NH}_{2}(7 \mathrm{mkm})$ served as the stationary phase, whereas a 75/25 v/v acetonitrile/water solution was used as the mobile phase. The flow rate was held constant at $100 \mathrm{~mL} / \mathrm{min}$ at 20 bar and $20{ }^{\circ} \mathrm{C}$. The wavelength of UV detection was maintained below $190 \mathrm{~nm}$. The concentration of the sodium salt of 2-keto-L-gulonic acid was varied from 0.01 to $20 \mathrm{mg} / \mathrm{mL}$, and the relative retention of the internal standard Xylite (10 $\mathrm{mg} / \mathrm{mL}$ ) to the sodium salt of 2-keto-L-gulonic acid was 3:1.

Characterization. The Pt content was obtained by X-ray fluorescence (XRF) measurements performed with a Zeiss Jena VRA-30 spectrometer (Mo anode, LiF crystal analyzer, and SZ 

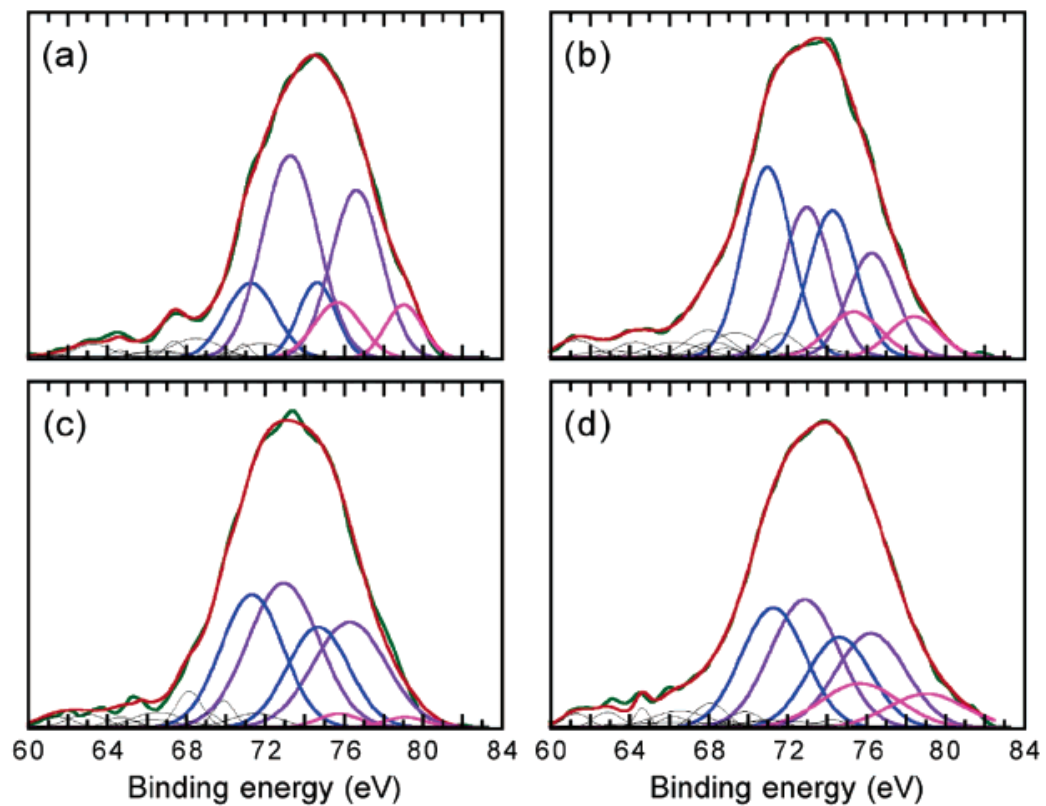

Figure 1. X-ray photoelectron spectra (green lines) of the following HPS nanocomposites: (a) MN-270-Pt, (b) MN-270-Pt-induction, (c) MN270-Pt-OX, and (d) MN-270-Pt-3OX. The Pt 5 $\mathrm{p}_{1 / 2}$ binding energy at $69 \mathrm{eV}$ and satellites from $\mathrm{MgK} \alpha_{3}$ and $\mathrm{MgK} \alpha_{4}$ at 8.4 and $10 \mathrm{eV}$ are shown as gray lines. The blue, violet, and magenta peaks denote the deconvolutions of $\mathrm{Pt}(0), \mathrm{Pt}(\mathrm{II})$, and $\mathrm{Pt}(\mathrm{IV})$ in $\mathrm{K}_{2} \mathrm{PtCl} \mathrm{l}_{6}$. The red lines represent the calculated spectra as the sum of all the deconvoluted peaks.

detector). Analyses were based on the $\mathrm{Co} \mathrm{K} \alpha$ line and a series of PS/Pt standards prepared by mixing $1 \mathrm{~g}$ of PS with $10-20$ $\mathrm{mg}$ of standard Pt compounds. The time of data acquisition was constant at $10 \mathrm{~s}$. Nitrogen sorption was conducted at the normal boiling point of liquid nitrogen using a Micromeritics Gemini instrument. Samples were degassed at $100{ }^{\circ} \mathrm{C}$ in vacuo for 12 $\mathrm{h}$ prior to analysis. X-ray photoelectron spectra were obtained using $\mathrm{Mg} \mathrm{K} \alpha(h v=1253.6 \mathrm{eV})$ monochromatized radiation with a modified ES-2403 spectrometer (provided by the Institute for Analytic Instrumentation of the Russian Academy of Sciences, St. Petersburg, Russia). The analyzer was operated at a pass electron energy of $100 \mathrm{eV}$. All data were acquired at an X-ray power of $100 \mathrm{~W}$ and an energy step of $0.1 \mathrm{eV}$. The electron-flood gun accessory was used so that the current of the total emitted electron flux from the flood-gun filament was adjustable from 0 to $100 \mathrm{~mA}$, with the optimum found to be 70 $\mathrm{mA}$ at an electron energy of $2 \mathrm{eV}$. Samples were allowed to outgas for 15-30 min before analysis and were sufficiently stable during examination. Data analysis was performed using a standard RFES-set with Resolver program. Identification of band components was achieved by assuming that the Pt $3 d_{5 / 2}$ and $3 \mathrm{~d}_{3 / 2}$ spin-orbit components (i) exhibit a Gaussian peak shape, (ii) are in 3:2 peak area ratio, and (iii) are separated by ca. $5 \mathrm{eV}$. Surface compositions were discerned by measuring the peak-area intensities of different Pt species.

$\mathrm{X}$-ray diffraction patterns were collected on a Scintag $\theta-\theta$ powder diffractometer. X-rays were generated with $\mathrm{Cu} \mathrm{K} \alpha$ radiation with a wavelength of $0.154 \mathrm{~nm}$. The step size of each run was maintained at $0.02^{\circ}$. Transmission electron microscopy was performed with a Zeiss EM902 electron spectroscopic microscope operated at $80 \mathrm{kV}$ and an energy loss of $0 \mathrm{eV}$. Insoluble HPS-Pt powders were embedded in epoxy resin and subsequently microtomed at ambient temperature. Images of the resulting thin sections (ca. $50 \mathrm{~nm}$ thick) were collected on plate negatives, digitized at $600 \mathrm{dpi}$, and analyzed with the Adobe Photoshop software package and the Scion Image Processing Toolkit. The ASAXS analysis was performed at the JUSIFA beamline $^{35}$ at HASYLAB, DESY (Hamburg, Germany), over an energy range in close proximity to the $\mathrm{L}_{\mathrm{III}}$-absorption edge
TABLE 1: Characteristics of the Pt-Containing Nanocomposites Prepared from MN-270

\begin{tabular}{|c|c|c|c|c|}
\hline material notation & Pt species & $\begin{array}{l}E^{a} \mathrm{Pt} 4 \mathrm{f}_{7 / 2} \\
( \pm 0.1 \mathrm{eV})\end{array}$ & $\% \operatorname{Pt}(i)$ & $\begin{array}{l}\text { BET surface } \\
\text { area }\left(\mathrm{m}^{2} / \mathrm{g}\right)\end{array}$ \\
\hline \multirow[t]{3}{*}{$\mathrm{MN}-270-\mathrm{Pt}$} & $\operatorname{Pt}(0)$ & 71.5 & 25.9 & \multirow[t]{3}{*}{781} \\
\hline & $\mathrm{Pt}(\mathrm{II})$ & 73.6 & 48.3 & \\
\hline & $\mathrm{Pt}(\mathrm{IV})$ & 75.7 & 25.8 & \\
\hline \multirow[t]{3}{*}{ MN-270- Pt-induction } & $\operatorname{Pt}(0)$ & 71.6 & 40.9 & \multirow[t]{3}{*}{325} \\
\hline & $\mathrm{Pt}(\mathrm{II})$ & 72.9 & 39.5 & \\
\hline & $\mathrm{Pt}(\mathrm{IV})$ & 75.7 & 19.6 & \\
\hline \multirow{3}{*}{$\mathrm{MN}-270-\mathrm{Pt}-\mathrm{OX}$} & $\operatorname{Pt}(0)$ & 71.4 & 41.8 & \multirow[t]{3}{*}{602} \\
\hline & $\mathrm{Pt}(\mathrm{II})$ & 72.9 & 36.8 & \\
\hline & $\mathrm{Pt}(\mathrm{IV})$ & 75.7 & 21.4 & \\
\hline \multirow[t]{3}{*}{ MN-270-Pt-3OX } & $\operatorname{Pt}(0)$ & 71.6 & 42.9 & \multirow[t]{3}{*}{542} \\
\hline & $\mathrm{Pt}(\mathrm{II})$ & 72.8 & 31.8 & \\
\hline & $\mathrm{Pt}(\mathrm{IV})$ & 75.7 & 25.2 & \\
\hline
\end{tabular}

of Pt (11.564 keV). Measurements were obtained with a twodimensional detector at two energies (11.484 and $11.562 \mathrm{keV})$. The energy dependence of small-angle scattering near the $\mathrm{L}_{\mathrm{III}}{ }^{-}$ absorption edge of $\mathrm{Pt}$ was used to differentiate the scattering due specifically to Pt-containing heterogeneities from that associated with, for instance, pores in the polymer matrix. Details pertaining to data analysis are provided in the following section.

\section{Results and Discussion}

Structure Analysis. As discussed elsewhere, ${ }^{36}$ incorporation of the THF solution containing platinic acid into microporous HPS results in partial reduction of the Pt(IV) species and the formation of $\mathrm{Pt}(\mathrm{II})$ complexes wherein ligands are the product of THF oxidation. Figure 1 presents XPS spectra (and their deconvoluted contributions) of Pt-containing MN-270 at different stages of the catalytic reaction. The fraction of each type of $\mathrm{Pt}$ species ascertained from the deconvolution analysis is listed in Table 1. In the present work, addition of the THF/ platinic acid solution to $\mathrm{MN}-270$ yields the unchanged $\mathrm{Pt}(\mathrm{IV})$ (25.8\%), as well as $\mathrm{Pt}(\mathrm{II})(48.3 \%)$ and even $\mathrm{Pt}(0)(25.9 \%)$. This analysis suggests that the chosen reaction conditions facilitate 

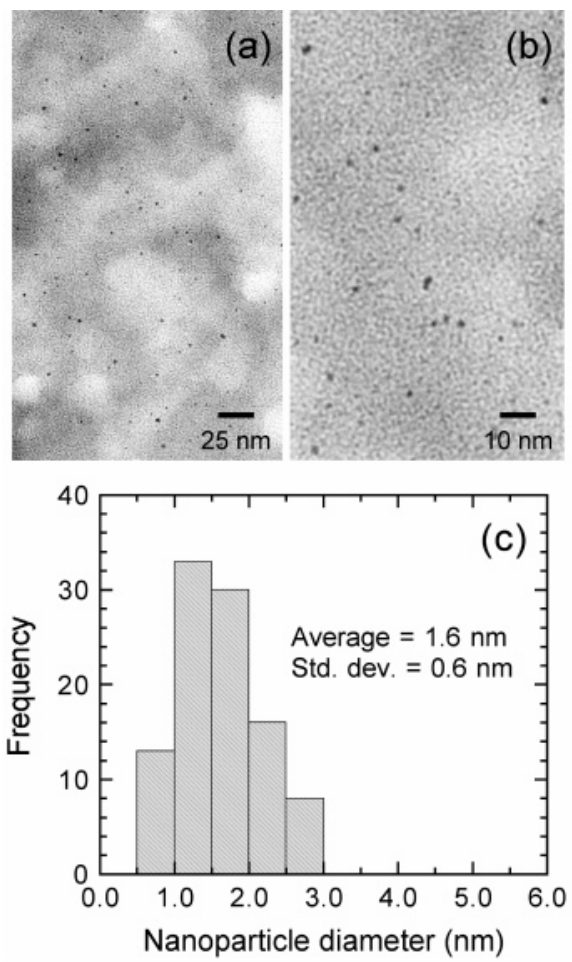

Figure 2. TEM images $(\mathrm{a}, \mathrm{b})$ and corresponding particle size histogram (c) of MN-270-Pt containing 1.72 wt \% Pt.

Pt reduction since no $\mathrm{Pt}(0)$ was previously detected ${ }^{36}$ in the microporous HPS after incorporation of platinic acid. Parts a and $b$ of Figure 2 are TEM images of the MN-270-Pt hybrid after initial incorporation of platinic acid. The material is observed to contain electron-dense nanoparticles with a diameter exceeding $0.7 \mathrm{~nm}$ (the estimated resolution of the microscope). The mean particle diameter calculated from a particle size histogram (Figure 2c) is $1.6 \mathrm{~nm}$, and the standard deviation is $0.6 \mathrm{~nm}$. Since HPS constitutes a highly hydrophobic matrix, the Pt species deposited from the platinic acid do not distribute molecularly within the HPS matrix but instead form well-defined clusters. The light background regions in this and subsequent TEM images represent the macropores alluded to earlier (the micropores are not resolved). The corresponding XRD pattern of this material is displayed in Figure $3 \mathrm{a}$ and confirms the absence of scattering from crystalline Pt. It does not, however, preclude the possibility of scattering from amorphous Pt. Last, it should be noted that the BET surface area of this Pt-modified HPS (see Table 1) is similar to that of the parent MN-270 homopolymer (provided in the Experimental Section), indicating that the incorporation of Pt species into the HPS matrix does not obstruct the micropores.

When this metal-polymer nanocomposite is used as a catalyst in the oxidation of L-sorbose to 2-keto-L-gulonic acid according to Scheme 1, L-sorbose is slowly consumed for the first $60 \mathrm{~min}$ of the reaction but the product (2-keto-L-gulonic acid) is not detected. This stage is referred to as the induction period, and the Pt-modified MN-270 isolated after this period is denoted MN-270-Pt-induction. According to the XPS spectra presented in Figure $1 \mathrm{~b}$ and the deconvolution results recorded in Table 1, this material contains a higher fraction of $\mathrm{Pt}(0)$ and a lower fraction of $\mathrm{Pt}(\mathrm{II})$ and $\mathrm{Pt}(\mathrm{IV})$. The XRD pattern of this material (cf. Figure $3 b$ ) shows peaks assigned only to the $\mathrm{Pt}(0)$ crystalline phase. ${ }^{39,40}$ The average $\operatorname{Pt}(0)$ crystallite size (calculated from the Scherrer equation for $2 \theta=39.8^{\circ}$ along the (111) projection) is $2.8 \mathrm{~nm}$. No peaks signifying ${ }^{41}$ the existence of $\mathrm{PtO}$ or $\mathrm{PtO}_{2}$ crystals are evident in Figure $3 \mathrm{~b}$, in which case the presence of $\mathrm{Pt}$ at higher oxidative states, such as $\mathrm{Pt}(\mathrm{II})$ and $\mathrm{Pt}(\mathrm{IV})$, in the XPS spectra of this sample are attributed to either (i) incomplete reduction by L-sorbose or (ii) the modification of nanoparticle surface atoms by 2-keto-L-gulonic acid. It should be recognized, however, that high binding energy values may also reflect charging of metal nanoparticles in a nonconductive polymer environment. ${ }^{42,43}$ In this case, the species comprising the nanoparticles would be expected to exhibit an increased oxidation state during analysis. Moreover, according to the results listed in Table 1, the BET surface area of this sample is substantially lower (by more than a factor of 2) than that of the MN-270-Pt material after incorporation of platinic acid, strongly suggesting that the micropores in the HPS matrix are impeded by the presence of reaction species (L-sorbose and 2-keto-Lgulonic acid).

The TEM images and corresponding particle size histogram presented in Figure 4 reveal that the Pt nanoparticle size and
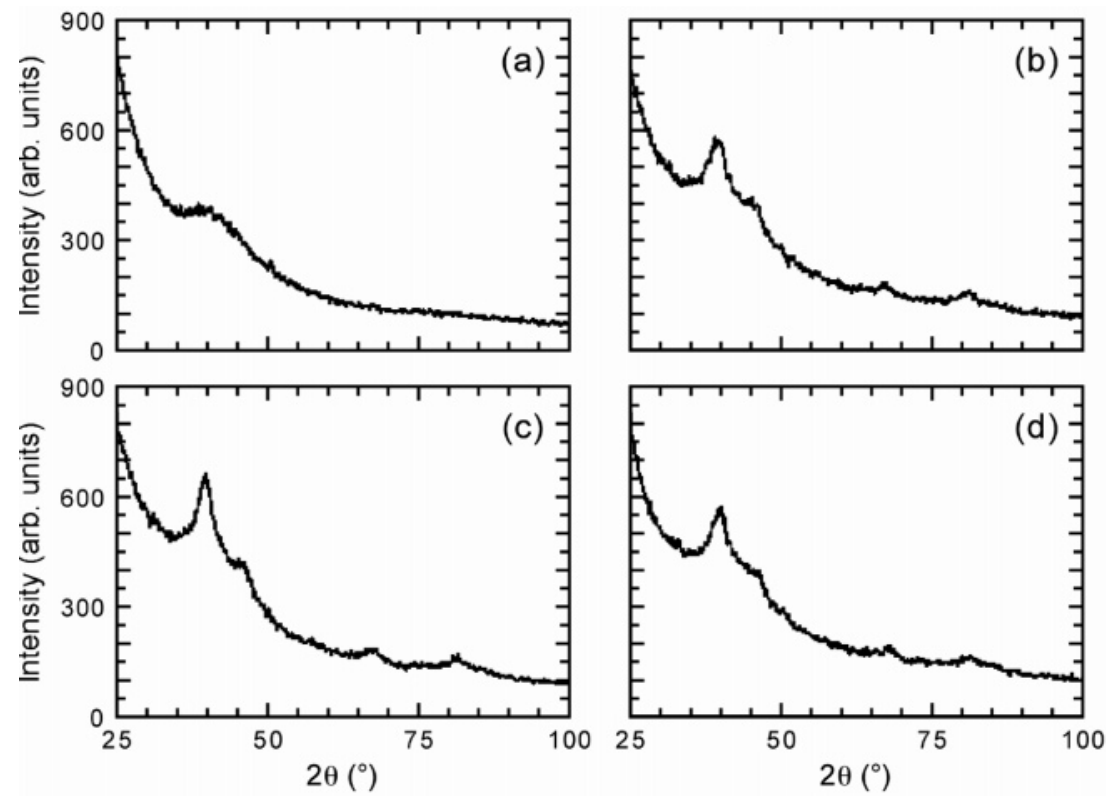

Figure 3. XRD patterns of the following HPS nanocomposites: (a) MN-270-Pt, (b) MN-270-Pt-induction, (c) MN-270-Pt-OX and (d) MN-270Pt-3OX. 

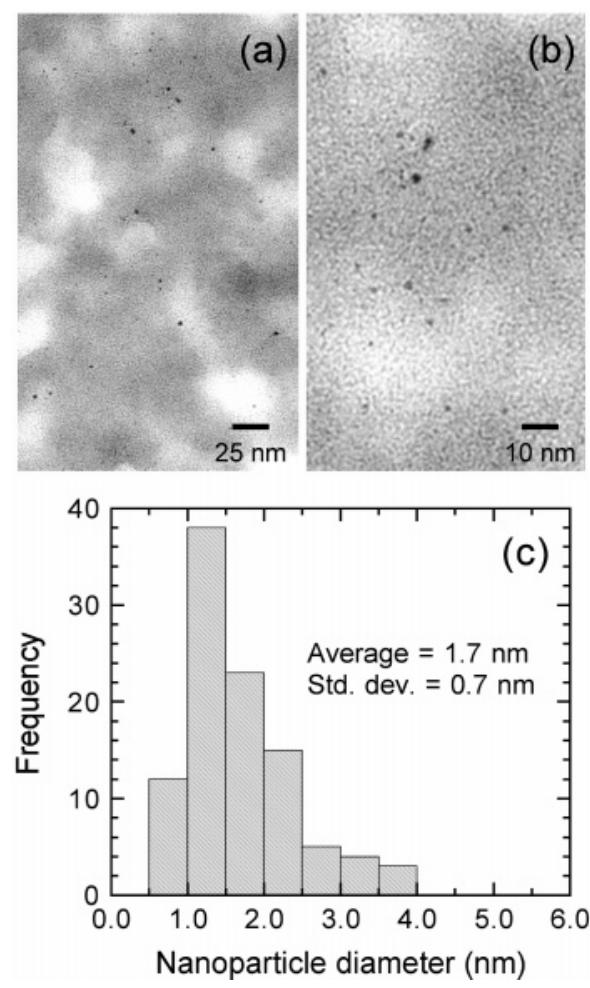

Figure 4. TEM images $(\mathrm{a}, \mathrm{b})$ and corresponding particle size histogram (c) of MN-270-Pt-induction (after the induction period in the catalytic oxidation of L-sorbose).

size distribution in the $\mathrm{MN}-270-\mathrm{Pt}$-induction material are surprisingly comparable to those in the initial $\mathrm{MN}-270-\mathrm{Pt}$ material. Despite the formation of slightly larger nanoparticles (measuring up to ca. $4 \mathrm{~nm}$ in diameter) following the induction period, such similarity strongly suggests that the mixed $\mathrm{Pt}(0 /$ II/IV) clusters serve as nuclei in the formation of $\operatorname{Pt}(0)$ nanoparticles and that the nanoparticle size is, for the most part, controlled by the size of the HPS micropores. The larger mean particle size deduced from the XRD pattern in Figure $3 \mathrm{~b}$ implies that the small Pt nanoparticles in Figure 4a,b may be amorphous. Recall that this possibility could not be discounted from the pattern acquired from the MN-270-Pt material. Reduction of $\mathrm{Pt}$ in $\mathrm{MN}-270-\mathrm{Pt}$ by L-sorbose during the induction period of the reaction seems to stabilize the nanoparticle surface. The HPS macropores, on the other hand, provide easy access of the reaction medium to the growing nanoparticles, thereby facilitating nanoparticle nucleation. In addition, oxidation products may likewise adsorb onto and help stabilize the surface of the growing Pt nanoparticles. Together, these molecular-scale events contribute to the apparent tight control over nanoparticle size. In contrast, access of L-sorbose to resident Pt species and the subsequent availability of 2-keto-L-gulonic acid (a possible nanoparticle stabilizer) are both hindered in fully microporous HPS by the small pore size. Diffusion-limiting considerations such as these are deemed responsible for the larger and more broadly distributed Pt nanoparticles observed ${ }^{33}$ after the induction period in HPS possessing only micropores.

Figures 5 and 6 are TEM images and corresponding particle size histograms of HPS/Pt nanocomposite catalysts isolated after one catalytic run (MN-270-Pt-OX, Figure 5) and three cycles (MN-270-Pt-3OX, Figure 6). With the exception that a relatively small population of nanoparticles appears to aggregate into nanoparticles measuring greater than $\sim 3.5 \mathrm{~nm}$ in diameter, the mean particle diameter calculated from both histograms remains unchanged. Thus, it follows that the catalytic oxidation examined here does not destabilize the Pt nanoparticles within the HPS
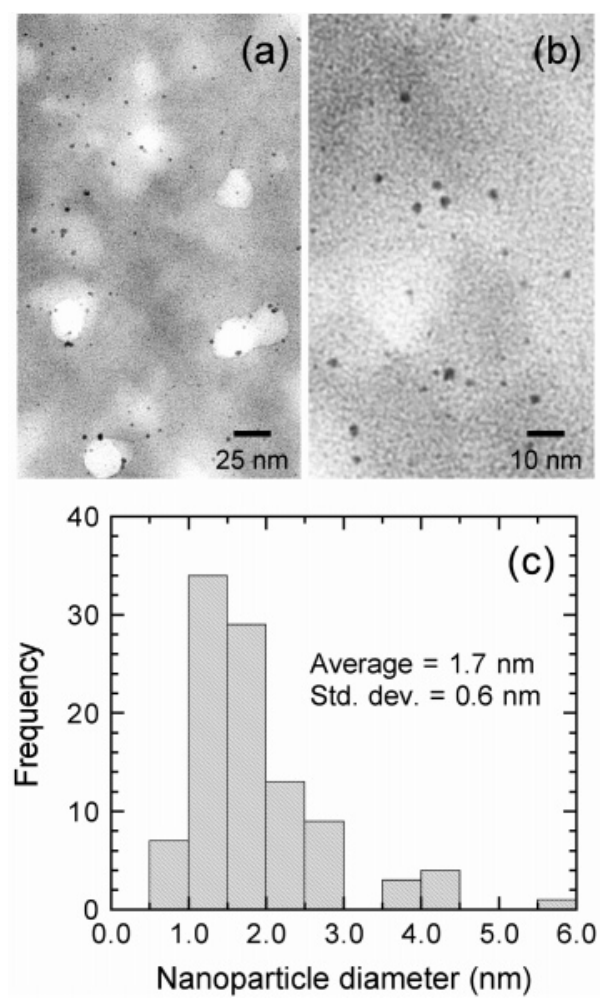

Figure 5. TEM images $(a, b)$ and corresponding particle size histogram (c) of MN-270-Pt-OX (after one completed cycle in the catalytic oxidation of L-sorbose).
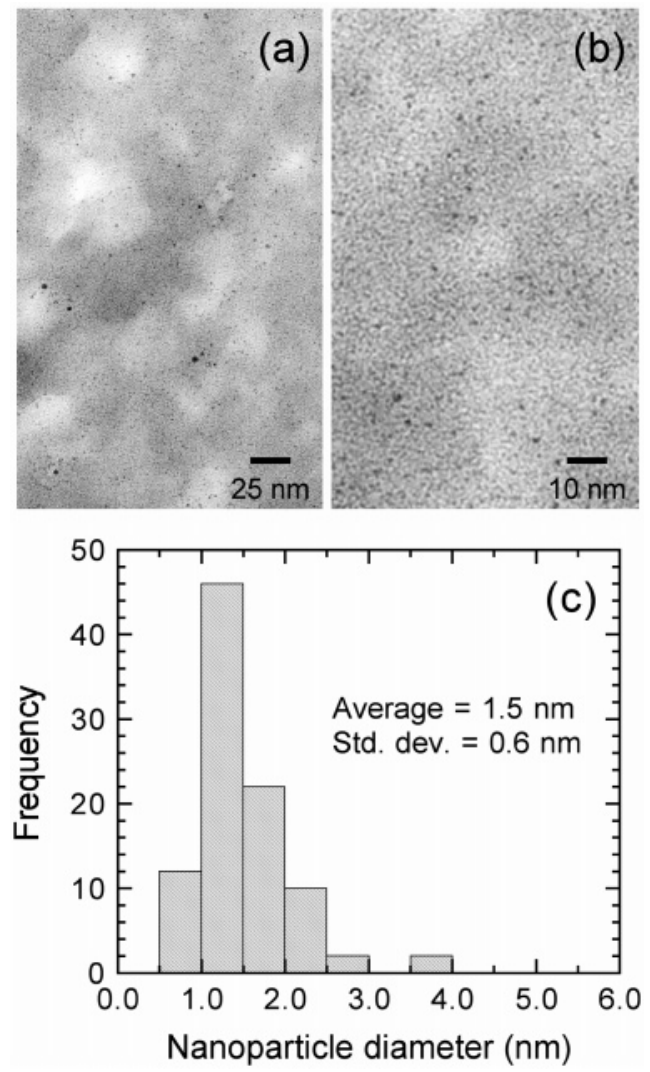

Figure 6. TEM images $(a, b)$ and corresponding particle size histogram (c) of MN-270-Pt-3OX (after three consecutive cycles in the catalytic oxidation of L-sorbose).

nanocomposite. It is particularly noteworthy that, after three consecutive catalytic runs, no further nanoparticle aggregation is observed. In fact, the relatively large nanoparticles $(>4 \mathrm{~nm}$ 

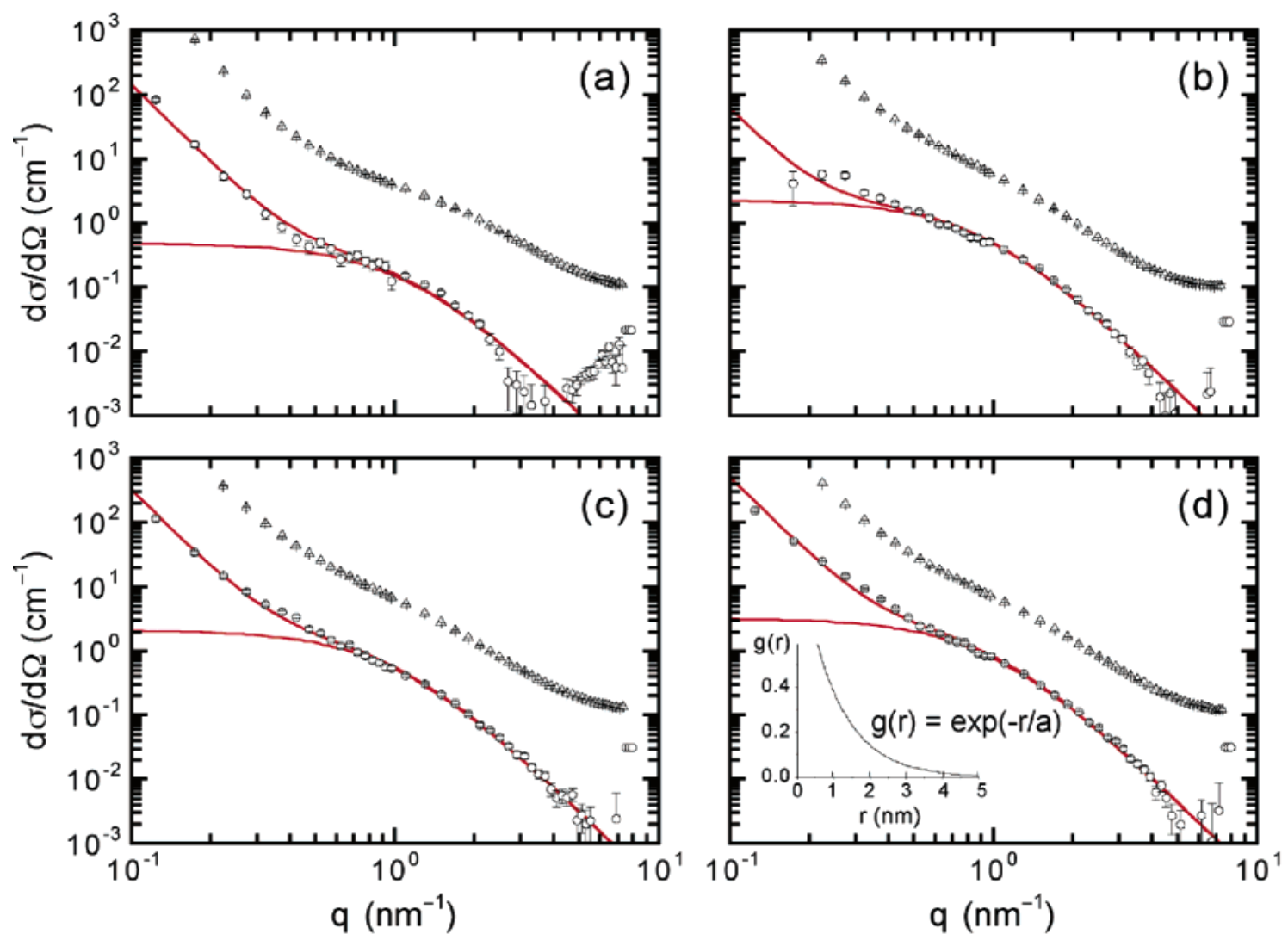

Figure 7. Small-angle X-ray scattering curves of the following HPS nanocomposites: (a) MN-270-Pt, (b) MN-270-Pt-induction, (c) MN-270-PtOX and (d) MN-270-Pt-3OX. The total scattering curves $(\triangle)$ are measured at $11.484 \mathrm{keV}$, while the separated scattering curves $(O)$ are obtained from measurements acquired at 11.484 and $11.562 \mathrm{keV}$. The solid lines are regressed fits to the model provided in eq 1 . The inset in (d) shows the correlation function of the model proposed by Debye et al. ${ }^{41}$ (see text).

in diameter) introduced after the first cycle are no longer detected by TEM after the third cycle. Although we recognize that this population of nanoparticles is small and may easily be overlooked during sampling, we cannot discount the possibility that these nanoparticles actually disperse in the presence of the reaction media so that the mean particle diameter decreases slightly. Results acquired from XRD are consistent with this scenario. The XRD patterns of MN-270-Pt-OX and MN-270Pt-3OX (cf. parts c and d, respectively, of Figure 3) exhibit reflexes identifying $\operatorname{Pt}(0)$ crystallites with mean diameters of 3.2 and $2.8 \mathrm{~nm}$, respectively. The XPS spectra collected from these nanocomposites after cycling (Figure 1c for MN-270-PtOX and Figure 1d for MN-270-Pt-3OX), as well as the deconvolution results (see Table 1), are virtually identical to those of the MN-270-Pt-induction material (Figure 1b), suggesting that the Pt nanoparticle composition does not change after the catalytic oxidation of L-sorbose. One property that does change upon catalytic oxidation is the BET surface area, which increases sharply from $325 \mathrm{~m}^{2} / \mathrm{g}$ after induction to $\sim 600 \mathrm{~m}^{2} / \mathrm{g}$ after just one catalytic run (included in Table 1). These results suggest that the HPS micropores become less obstructed relative to those following the induction period when the reaction is complete.

The enhanced stability of the Pt nanoparticles within MN270 is indeed surprising. In the presence of macropores, it is reasonable to expect greater aggregation of the nanoparticles during catalytic cycling. Such stability can be explained in terms of the following mechanism. The Pt clusters formed in MN270 after incorporation of platinic acid preferentially locate inside (without obstructing) the micropores, not at the macropore interfaces. If they did reside at the macropore interfaces, we suspect that the nanoparticle stability might be sorely compromised after consecutive exposure to the reaction medium. Such preferential positioning of the Pt clusters would most likely occur due to their hydrophobic shell that develops because of the presence of organic ligands in the $\mathrm{Pt}(\mathrm{II})$ complex. $^{36}$ Subsequent Pt nanoparticle nucleation and growth occur exclusively in the micropores, which effectively serve to restrict nanoparticle size. The small fraction of Pt nanoparticles with diameters larger $(2.5-4.0 \mathrm{~nm})$ than the average nanopore diameter $(\sim 1.5 \mathrm{~nm})$ may result from either (i) clusters or nanoparticles that reside in pore defects or junctions within the native HPS matrix or (ii) neighboring nanoparticles that aggregate as the HPS matrix swells in the reaction medium. It is possible that such aggregation may occur only upon initial exposure to the reaction medium. While it would be intuitive to expect that the extent of such aggregation would increase upon repeated exposure, the TEM and XRD results reported here indicate that consecutive exposure of the Pt nanoparticles to the reaction medium may actually serve to reduce the mean nanoparticle size.

To glean insight into the overall structure of these metal/ polymer nanocomposites, all four MN-270-Pt materials acquired at different stages of the L-sorbose oxidation reaction have been investigated by ASAXS (cf. Figure 7). The total scattering curve (measured at $11.484 \mathrm{keV}$ ) and the separated scattering curve (obtained from 11.484 and $11.562 \mathrm{keV}$ ) are provided for the MN-270-Pt (Figure 7a), the MN-270-Pt-induction (Figure 7b), the MN-270-Pt-OX (Figure 7c), and the MN-270-Pt-3OX (Figure 7d) materials. Each specimen clearly reveals significant contributions from nonuniformly distributed Pt within the polymer matrix. The solid line through each separated scattering 
TABLE 2: Structure Parameters and Compositions Extracted from the ASAXS Analysis

\begin{tabular}{|c|c|c|c|c|c|c|}
\hline material notation & $a(\mathrm{~nm})$ & $b\left(\mathrm{~cm}^{-1} / \mathrm{nm}^{3}\right)$ & $c\left(\mathrm{~cm}^{-1} / \mathrm{nm}^{4}\right)$ & $\phi(\%)$ & $w_{\phi}^{a}(\%)$ & $w_{\mathrm{ea}}^{b}(\%)$ \\
\hline $\mathrm{MN}-270-\mathrm{Pt}^{c}$ & $0.89(3)$ & $0.74(3)$ & $0.015(1)$ & & & 1.72 \\
\hline MN-270-Pt-induction & $1.07(2)$ & $1.97(4)$ & $0.006(1)$ & 0.025 & 1.04 & 2.97 \\
\hline MN-270-Pt-OX & $0.99(2)$ & $2.31(4)$ & $0.035(1)$ & 0.030 & 1.22 & 3.24 \\
\hline MN-270-Pt-3OX & $1.02(1)$ & $3.09(4)$ & $0.050(1)$ & 0.040 & 1.62 & 3.16 \\
\hline
\end{tabular}

${ }^{a}$ The weight percent of Pt calculated from the volume fraction. ${ }^{b}$ The weight percent of Pt measured from elemental analysis. ${ }^{c}$ The volume fraction and weight percent of MN-270-Pt are not given, since it contains no detectable $\mathrm{Pt}(0)$ phase.

curves is a regression of a model introduced by Debye et al. ${ }^{44}$ with an additional $\mathrm{q}^{-4}$ term describing large inhomogeneities (eq 1):

$$
\Delta \frac{\mathrm{d} \sigma^{\mathrm{tot}}}{\mathrm{d} \Omega}\left(q, E_{1}, E_{2}\right)=\frac{b a^{3}}{\left(1+a^{2} q^{2}\right)^{2}}+\frac{c}{q^{4}}
$$

Here, $\Delta \mathrm{d} \sigma^{\mathrm{tot}} / \mathrm{d} \Omega$ is the separated scattering intensity obtained from the total scattering curves measured at energies $E_{1}$ and $E_{2}, q(=4 \pi / \lambda \sin \theta)$ is the scattering vector with the wavelength $\lambda$ and the scattering angle $2 \theta, a$ is the correlation length, and $b$ and $c$ are adjustable parameters. ${ }^{45}$ The model represents the small-angle scattering of inhomogeneities with fully random shape, size and distribution. The correlation function with the correlation length $a$ is shown for example in the inset of Figure $7 \mathrm{~d}$, and the related scattering curve obtained from this function (without the $q^{-4}$ term) lies normal to the ordinate. Table 2 summarizes the results obtained from fitting eq 1 to the separated scattering curves in Figure 7. The correlation lengths approximate the Pt nanoparticle sizes and agree favorably with the sizes measured by TEM.

The volume fraction of Pt nanoparticles in each specimen can likewise be deduced from the parameter $b$ if the electron density contrast of the particles relative to the HPS matrix is known. Assuming that the nanoparticles are $\mathrm{Pt}(0)$ (with a mass density of $\left.21.41 \mathrm{~g} / \mathrm{cm}^{3}\right)$, the volume fractions $(\phi)$ have been calculated according to the methodology presented earlier. ${ }^{45}$ From these volume fractions, the weight percents $\left(w_{\phi}\right)$ of Pt in the four materials have been calculated and compared in Table 2 to results obtained from elemental analysis $\left(w_{\mathrm{ea}}\right)$. The $\mathrm{Pt}$ compositions extracted from the ASAXS model lie between 35 and $51 \%$ of the compositions acquired from elemental analysis. Two plausible explanations are offered for the "missing" Pt in the ASAXS analysis: (i) the larger structures, which require the $q^{-4}$ term in eq 1 to fit the separated scattering curves, are required to contain $\mathrm{Pt}$, and (ii) the electron density contrast introduced into the calculation of the volume fractions from the correlation model may be smaller than anticipated due to the presence of $\mathrm{Pt}$ oxides. The first explanation is confirmed by TEM images that illustrate the presence of macropores, which are expected to be partially decorated with Pt due to Pt species located in adjacent micropores. Moreover, the oxidation state of the Pt is known from the XPS spectra displayed in Figure 1 to vary (from 0 to IV) due to chemical modification in the presence of the reaction media (or possible charging during analysis). Since these two considerations are not mutually exclusive, a combination of both explanations is most likely applicable in resolving the apparent discrepancy in Pt compositions evident in Table 2.

Property Analysis. The optimal conditions for L-sorbose oxidation with MN-270-Pt have been identified by systematically varying the following reaction parameters: (i) catalyst concentration $\left(C_{\mathrm{c}}\right)$ from 0.001 to $0.010 \mathrm{M} \mathrm{Pt}$, (ii) L-sorbose concentration $\left(C_{0}\right)$ from 0.05 to $0.15 \mathrm{M}$, (iii) $\mathrm{NaHCO}_{3}$ concentration $\left(C_{\mathrm{NaHCO}_{3}}\right)$ from 0.05 to $0.15 \mathrm{M}$, (iv) reaction temperature (T) from 60 to $80{ }^{\circ} \mathrm{C}$, (v) reaction time $(t)$ from 180 to 220

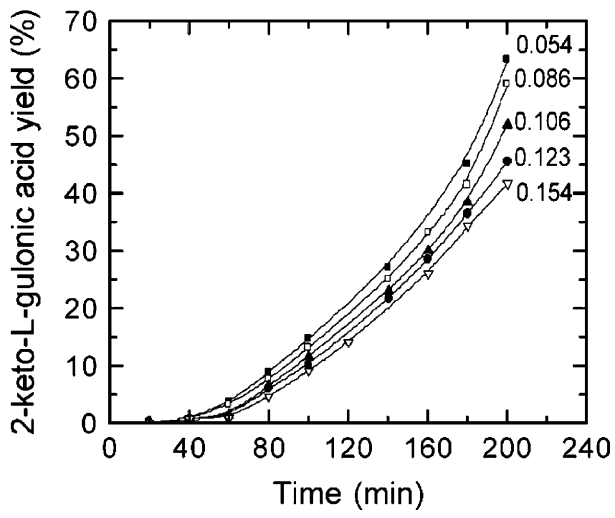

Figure 8. Dependence of 2-keto-L-gulonic acid yield on reaction time in the presence of MN-270-Pt at different L-sorbose molar concentrations (displayed in the figure). Pt content is $1.72 \mathrm{wt} \%$.

min, (vi) oxygen flow rate $\left(V_{\mathrm{O}}\right)$ from $7.5 \times 10^{-6}$ to $20 \times 10^{-6}$ $\mathrm{m}^{3} / \mathrm{s}$, and (vii) stirring rate from 200 to $1000 \mathrm{rpm}$. The highest selectivity obtained at $100 \%$ L-sorbose conversion during the course of this study is $96.8 \%$. It is achieved with the MN-270Pt nanocomposite under the following set of reaction conditions: $C_{\mathrm{c}}=0.002 \mathrm{M} \mathrm{Pt}, C_{0}=0.106 \mathrm{M}, C_{\mathrm{NaHCO}_{3}}=0.106 \mathrm{M}$, $T=70{ }^{\circ} \mathrm{C}, V_{0}=14 \times 10^{-6} \mathrm{~m}^{3} / \mathrm{s}$, stirring rate $=1000 \mathrm{rpm}$, and $\mathrm{NaHCO}_{3}$ added continuously for $180 \mathrm{~min}$. The kinetic characteristics of selective L-sorbose oxidation with MN-270$\mathrm{Pt}$ have been ascertained by adjusting the substrate/catalyst concentration ratio $\left(\mathrm{C}_{0} / \mathrm{C}_{\mathrm{c}}\right)$ from 27.0 to $77.0 \mathrm{~mol} / \mathrm{mol} \mathrm{Pt}$ at a constant $\mathrm{O}_{2}$ flow rate of $14 \times 10^{-6} \mathrm{~m}^{3} / \mathrm{s}$ and a stirring rate of $1000 \mathrm{rpm}$ at $70{ }^{\circ} \mathrm{C}$. For this analysis, the reaction medium was charged with a single shot of the alkaline agent (in an equimolar amount with respect to the L-sorbose) before the beginning of the reaction. The dependence of L-sorbose conversion on reaction time is presented in Figure 8 and demonstrates that an increase in $C_{0}$ at constant $C_{\mathrm{c}}$ promotes an increase in reaction time $(t)$, which is typical for most catalysts. These kinetic curves also verify the existence of a $60 \mathrm{~min}$ induction period, during which time 2-keto-L-gulonic acid yield is negligible. Comparison of this induction period with results presented in our previous study $^{36}$ of fully microporous HPS reveals a substantial decrease in induction time from 100 to $60 \mathrm{~min}$ for the micro/macroporous $\mathrm{MN}-270-\mathrm{Pt}$ nanocomposite. This reduction is attributed to presence of macropores in the HPS matrix and, hence, facilitated reactant and product transport.

The effect of temperature on the kinetics of L-sorbose oxidation has also been investigated from 60 to $80{ }^{\circ} \mathrm{C}$ at the same conditions employed above, and the resulting data (see Table 3) establish that the rate of L-sorbose oxidation increases with increasing temperature. At the highest selectivity and conversion of the MN-270-Pt nanocomposite measured in this work (96.8\% selectivity at $100 \%$ conversion), the value of the catalytic activity is $2.5 \times 10^{-3} \mathrm{~mol} / \mathrm{mol} \mathrm{Pt}-\mathrm{s}$, which corresponds to a $4.6 \times$ increase compared to the fully microporous HPS/Pt analogue $^{36}$ and an even greater increase relative to other oxidation catalysts. ${ }^{46}$ This enhanced catalytic activity is likewise attributed to the presence of a hierarchical pore morphology. 
TABLE 3: Effect of Temperature on the Direct Oxidation of L-Sorbose with $\mathrm{MN}-270-\mathrm{Pt}^{a}$

\begin{tabular}{lrrrrr}
\hline$T\left({ }^{\circ} \mathrm{C}\right)$ & \multicolumn{1}{c}{60} & \multicolumn{1}{c}{65} & \multicolumn{1}{c}{70} & \multicolumn{1}{c}{75} & \multicolumn{1}{l}{80} \\
selectivity $(\%)$ & 81.3 & 88.7 & 96.8 & 84.8 & 75.1 \\
activity $\times 10^{3}(\mathrm{~mol} / \mathrm{mol} \mathrm{Pt-s})$ & 2.1 & 2.3 & 2.5 & 2.9 & 3.1
\end{tabular}

${ }^{a}$ Reaction conditions: $V_{0}=14 \times 10^{-6} \mathrm{~m}^{3} / \mathrm{s} ; \mathrm{NaHCO}_{3}$ is charged once before the beginning of the reaction; $C_{0}=0.106 \mathrm{M} ; C_{0} / C_{\mathrm{c}}=$ $53.6 \mathrm{~mol} / \mathrm{mol} \mathrm{Pt} ; t=200 \mathrm{~min}$.

TABLE 4: Activity and Selectivity of HPS Nanocomposites after Consecutive Catalytic Runs ${ }^{a}$

\begin{tabular}{lcc}
\hline \multicolumn{1}{c}{ nanocomposite catalyst } & $\begin{array}{c}\text { activity } \times 10^{3} \\
(\mathrm{~mol} / \mathrm{mol} \mathrm{Pt}-\mathrm{s})\end{array}$ & $\begin{array}{c}\text { selectivity } \\
(\%)\end{array}$ \\
\hline MN-270-Pt-OX & 2.5 & 96.8 \\
MN-270-Pt-3OX & 2.4 & 97.2 \\
microporous HPS/Pt (OX) & 0.54 & 95.7 \\
microporous HPS/Pt (3OX) & 0.53 & 95.0
\end{tabular}

$a$ The reactions are conducted under the same set of conditions listed in Table 3 at $70{ }^{\circ} \mathrm{C}$.

The temperature dependence of L-sorbose oxidation can be further analyzed if it obeys Arrhenius behavior. To establish the validity of this relationship, we have measured the reaction time yielding $10 \%$ L-sorbose conversion $\left(\tau_{0.1}\right)$. Regression of these data as a function of reciprocal temperature yields an apparent activation energy of $35 \pm 3 \mathrm{~kJ} / \mathrm{mol}$ for the $\mathrm{MN}-270$ $\mathrm{Pt}$ nanocomposite. This value compares well with $39 \pm 3 \mathrm{~kJ} /$ mol previously measured ${ }^{36}$ for the fully microporous HPS/Pt material. Since the activation energies of both catalysts are similar but the catalytic activities differ markedly, we have calculated a frequency factor from the ordinate intercept of the Arrhenius analysis to provide a measure of the number of collisions, or active centers, on the catalyst surface. The resulting frequency factor of MN-270-Pt is calculated to be $3.2 \times 10^{6}$, which is almost 2 orders of magnitude greater than that of the fully microporous HPS/Pt nanocomposite $\left(2.5 \times 10^{4}\right){ }^{36}$ This marked improvement in performance again reflects the presence of macropores and the greater accessibility of Pt nanoparticles to the reaction substrate. Table 4 provides a comparison of the catalytic activity and selectivity of the MN-270-Pt and fully microporous HPS/Pt nanocomposites after the first and third catalytic runs and confirms that the catalytic activity remains virtually constant, while the selectivity changes only slightly, in both materials. The unusually high stability, activity and selectivity of the Pt-modified MN-270 nanocomposite clearly make this material a promising catalyst for Pt-catalyzed reactions.

\section{Conclusions}

The formation of Pt nanoparticles within a micro/macroporous HPS matrix has been investigated in regard to the direct catalytic oxidation of L-sorbose to 2-keto-L-gulonic acid. In addition to studying the kinetics of this reaction, the structural characteristics of the nanoparticles have been examined at different stages of the reaction using a combination of XPS, TEM, XRD, and ASAXS. Incorporation of a THF solution containing platinic acid into the HPS matrix initially results in the formation of clusters containing Pt species of mixed valence states. The nanocomposite isolated from the reaction mixture after the reaction induction period reveals the formation of Pt nanoparticles of comparable size. Since further reaction (for $200 \mathrm{~min}$ ) to $100 \%$ L-sorbose conversion with the same catalyst does not change the nanoparticle size or structure, it may be concluded that the catalytically active Pt species form after the induction period. Shortening of the induction period from $100 \mathrm{~min}$ for fully microporous HPS to $60 \mathrm{~min}$ for the HPS with hierarchical porosity is attributed to the presence of macropores and facilitated L-sorbose transport. The corresponding $4.6 \times$ increase in catalytic activity relative to fully microporous catalyst is further credited to the macropores and the improved accessibility of the Pt nanoparticles to the reaction medium. The surprisingly high catalytic activity and selectivity, as well as stability after three catalytic runs, render this Pt-modified HPS nanocomposite with both micro- and macroporosity a robust hybrid material for Pt-catalyzed reactions, such as the direct oxidation of L-sorbose. The superb control over nanoparticle size and stability afforded by the micro/macroporous HPS matrix further indicate that HPS can serve as a platform for the development of novel metal/polymer nanocomposites to be used in emergent technologies requiring high accessibility to the surfaces of nearly monodisperse nanoparticles.

Acknowledgment. This work has been supported, in part, by the NATO Science for Peace Program (Grant No. SfP974173), the Russian Foundation for Basic Research (Grant No. 04-03-32928), and SINTEF Materials Technology.

\section{References and Notes}

(1) Heffels, W.; Friedrich, J.; Darribere, C.; Teisen, J.; Interewicz, K.; Bastiaansen, C.; Caseri, W.; Smith, P. Rec. Res. Devel. Macromol. Res. 1977, 2, 143 .

(2) Ellsworth, M. W.; Gin, D. L. Polymer News 1999, 24, 331.

(3) Gangopadhyay, R.; De, A. Chem. Mater. 2000, 12, 608. 303.

(4) Carotenuto, G.; Nicolais, L. Rec. Res. Devel. Mater. Sci. 2002, 3 ,

(5) Brayner, R.; Viau, G.; Bozon-Verduraz, F. J. Mol. Catal. A 2002, $182-183,227$.

(6) Liu, C.; Xu, Y.; Liao, S.; Yu, D. J. Mol. Catal. A 2000, 157, 253.

(7) Drelinkiewicz, A.; Hasik, M.; Kloc, M. J. Catal. 1999, 186, 123.

(8) Okitsu, K.; Yue, A.; Tanabe, S.; Matsumoto, H. Chem. Mater. 2000, 12,3006 .

(9) Horvath, A.; Beck, A.; Sarkany, A.; Guczi, L. Solid State Ionics 2002, 148, 219.

(10) Sanchez, C.; de Soler-Illia, G. J.; Ribot, F.; Lalot, T.; Mayer, C. R.; Cabuil, V. Chem. Mater. 2001, 13, 3061.

(11) Sankaran, V.; Cummins, C. C.; Schrock, R. R.; Cohen, R. E.; Silbey, R. J. J. Am. Chem. Soc. 1990, 112, 6858.

(12) Ellsworth, M. W.; Gin, D. L. Polymer News 1999, 24, 331.

(13) Chan, Y. N. C.; Schrock, R. R.; Cohen, R. E. J. Am. Chem. Soc. 1992, 114, 7295 .

(14) Chan, Y. N. C.; Craig, G. S. W.; Schrock, R. R.; Cohen, R. E. Chem. Mater. 1992, 4, 885.

(15) Hashimoto, T.; Harada, M.; Sakamoto, N. Macromolecules 1999, 32,6867 .

(16) Antonietti, M.; Wenz, E.; Bronstein, L.; Seregina, M. Adv. Mater. 1995, 7, 1000.

(17) Spatz, J. P.; Roescher, A.; Möller, M. Adv. Mater. 1996, 8, 337.

(18) Moffitt, M.; McMahon, L.; Pessel, V.; Eisenberg, A. Chem. Mater. 1995, 7, 1185.

(19) Zhao, M.; Crooks, R. M. Adv. Mater. 1999, 11, 217.

(20) Bronstein, L. M. Top. Curr. Chem 2003, 226, 55

(21) Shenhar, R.; Rotello, V. M. Acc. Chem. Res. 2003, 36, 549.

(22) Galow, T. H.; Drechsler, U.; Hanson, J. A.; Rotello, V. M. Chem. Commun. 2002, 1076.

(23) Miinea, L. A.; Sessions, L. B.; Ericson, K. D.; Glueck, D. S.; Grubbs, R. B. Polym. Preprints 2003, 44 (2), 214.

(24) Hulteen, J. C.; Martin, C. R. In Nanoparticles and Nanostructured Films; Fendler, J. H., Ed.; Wiley-VCH Verlag: Weinheim, 1998; p 235.

(25) Budd, P. M.; Makhseed, S. M.; Ghanem, B. S.; Msayib, K. J.; Tattershall, C. E.; McKeown, N. B. Mater. Today 2004, 40.

(26) Krause, B.; Koops, G.-H.; van der Vegt, N. F. A.; Wessling, M.; Wübbenhorst, M.; van Turnhout, J. Adv. Mater. 2002, 14.

(27) Plyuto, Y.; Berquer, J.-M.; Jacquiod, C.; Ricolleau, C. Chem. Commun. 1999, 1653.

(28) Bronstein, L. M.; Polarz, S.; Smarsly, B.; Antonietti, M. Adv. Mater. 2001, 13, 1333. 
(29) Carter, K. R.; DiPietro, R. A.; Sanchez, M. I.; Swanson, S. A. Chem. Mater. 2001, 13, 213.

(30) Ellsworth, M. W.; Gin, D. L. Polym. News 1999, 24, 331.

(31) Nikonorova, N. I.; Trofimchuk, E. S.; Elkin, P. G.; Belova, N. E.; Fanchenko, S. S.; Volynskii, A. L.; Bakeev, N. F. Vysokomol. Soedin., Ser. A Ser. B 2002, 44, 1185.

(32) Lu, Z.; Liu, G.; Phillips, H.; Hill, J. M.; Chang, J.; Kydd, R. A. Nanoletters 2001, 1, 683.

(33) Davankov, V. A.; Tsyurupa, M. P. React. Polym. 1990, 13, 27.

(34) Tsyurupa, M. P.; Davankov, V. A. J. Polym. Sci.: Polym. Chem. Ed. 1980, 18, 1399

(35) Sidorov, S. N.; Bronstein, L. M.; Davankov, V. A.; Tsyurupa, M. P.; Solodovnikov, S. P.; Valetsky, P. M.; Wilder, E. A.; Spontak, R. J. Chem. Mater. 1999, 11, 3210.

(36) Sidorov, S. N.; Volkov, I. V.; Davankov, V. A.; Tsyurupa, M. P.; Valetsky, P. M.; Bronstein, L. M.; Karlinsey, R.; Zwanziger, J. W.; Matveeva, V. G.; Sulman, E. M.; Lakina, N. V.; Wilder, E. A.; Spontak, R. J. J. Am. Chem. Soc. 2001, 123, 10502.

(37) Newalkar, B. L.; Komarneni, S. Chem. Mater. 2001, 13, 4573.

(38) Available at the URL http://www.purolite.com/.
(39) Bronstein, L. M.; Chernyshov, D. M.; Karlinsey, R.; Zwanziger, J. W.; Matveeva, V. G.; Sulman, E. M.; Demidenko, G. N.; Hentze, H.-P. Antonietti, M. Chem. Mater. 2003, 15, 2623.

(40) Arico, A. S.; Kim, H.; Shukla, A. K.; Ravikumar, M. K.; Antonucci V.; Giordano, N. Electrochim. Acta 1994, 39, 691.

(41) McBride, J. R.; Graham, G. W.; Peters, C. R.; Weber, W. H. J. Appl. Phys. 1991, 69, 1596.

(42) Wagner, C.; Riggs, W.; Davis, L.; Mullenberg, G. Handbook of $X$-ray Photoelectron Spectroscopy; Perkin-Elmer Corp.: Minneapolis, MN, 1978.

(43) Bronstein, L. M.; Chernyshov, D. M.; Volkov, I. O.; Ezernitskaya, M. G.; Valetsky, P. M.; Matveeva, V. G.; Sulman, E. M. J. Catal. 2000, 196, 302.

(44) Debye, P.; Anderson, H. R.; Brumberger, H. J. Appl. Phys. 1957, 28,679 . 181.

(45) Goerigk, G.; Williamson, D. L. J. Non-Cryst. Solids 2001, 281,

(46) Broennimann, C.; Bodnar, Z.; Aeschimann, R.; Mallat, T.; Baiker, A. J. Catal. 1996, 161, 720 . 